\title{
Retraction Note: Human cathelicidin LL-37 enhance the antibiofilm effect of EGCG on Streptococcus mutans
}

Yi-jie Guo $^{1,3^{*}+}$, Bo Zhang ${ }^{1,2+}$, Xue-song Feng ${ }^{1,3}$, Hui-xun Ren ${ }^{1,3}$ and Ji-ru Xu ${ }^{1,3^{*}}$

\section{Retraction}

The authors are retracting this article [1] because they do not have ownership of the data they report. A formal investigation by Tohoku University has concluded that the data reported in this article are the sole property of Tohoku University and have been reported in Bai et al. [2]. All authors agree with this retraction.

\footnotetext{
Author details

'Department of Pathogenic Microbiology and Immunology, School of Basic Medical Sciences, Xi'an Jiaotong University, Yanta West Road No.76, Xi'an 710061, ShaanXi, China. ${ }^{2}$ Clinical Laboratory, AnKang City Central Hospital, Jinzhou South Road No.85, AnKang 725000, ShaanXi, China. ${ }^{3}$ Key Laboratory of Environment and Genes Related to Diseases, Ministry of Education of China, Xi'an Jiaotong University, Yanta West Road No.76, Xi'an 710061, ShaanXi, China.
}

Received: 15 June 2017 Accepted: 16 June 2017

Published online: 26 June 2017

\section{References}

1. Guo Y-J, Zhang B, Feng X-S, Hui-xun Ren H-X, Xu J-R. Human cathelicidin LL-37 enhance the antibiofilm effect of EGCG on Streptococcus mutans. BMC Oral Health. 2016;16:101. doi:10.1186/s12903-016-0292-y.

2. Bai L, Takagi S, Ando T, Yoneyama H, Ito K, Mizugai H, Isogai E.

Antimicrobial activity of tea catechin against canine oral bacteria and the functional mechanisms. J Vet Med. 2016;78(9):1439-45.

\footnotetext{
*Correspondence: yijie_guo@mail.xjtu.edu.cn; xujiru@mail.xjtu.edu.cn

The online version of the original article can be found under doi:10.1186/ s12903-016-0292-y.

${ }^{\dagger}$ Equal contributors

'Department of Pathogenic Microbiology and Immunology, School of Basic Medical Sciences, Xi'an Jiaotong University, Yanta West Road No.76, Xi'an

710061, ShaanXi, China
} 